\title{
Lactobacillus and Its Probiotic Role in the Digestive and Nutritional Processes of Pigs: A Review
}

\section{Lactobacillus y su Papel Probiotico en el Pro- ceso Digestivo y Nutricional de Los Cerdos. Una Revision}

I International Seminar of Livestock and Agroindustrial Production ESPOCH 2020

Corresponding Author:

L. Tello

luis.tellof@espoch.edu.ec

Published: 2 September 2021

Production and Hosting by

Knowledge E

(c) L. Tello et al. This article is distributed under the terms of the Creative Commons Attribution License, which permits unrestricted use and redistribution provided that the original author and source are credited.
S OPEN ACCESS
L. Tello ${ }^{1}$, L. Flores ${ }^{2}$, J. Usca ${ }^{2}$, and I. Moreno ${ }^{3}$

'Laboratorio de Ciencias Biológicas, Facultad de Ciencias Pecuarias; Escuela Superior Politécnica de Chimborazo, Riobamba, Ecuador

${ }^{2}$ Docente, Facultad de Ciencias Pecuarias; Escuela Superior Politécnica de Chimborazo, Riobamba, Ecuador

${ }^{3}$ Laboratorio de Anatomía y Fisiología, Facultad de Ciencias Pecuarias, Escuela Superior Politécnica de Chimborazo, Riobamba, Ecuador

\section{Abstract}

The activity of a probiotic is dependent on the strain's ability to confer health benefits to the host through oral consumption of viable cells. The main objective of this study was to carry out a review about the importance of Lactobacillus and its probiotic role in the digestive and nutritional processes of pigs. The study focused on seven strains of Lactobacillus and their varied uses in feeding different categories of pigs. We obtained a clear conceptualization of Lactobacillus' effect on the immune system, its genome and how it is used in probiotic drinks. Based on the results, we can conclude that the use of Lactobacillus improves swine health and nutrition, making it a viable alternative to replace the indiscriminate use of antibiotics as growth promoters.

Keywords: probiotics, antibiotics, digestive process, endogenous species, pig categories.

\section{Resumen}

La habilidad de un probiótico está en dependencia de la capacidad de la cepa de conferir beneficios de salud al hospedador mediante el consuno oral de células viables. El presente trabajo tuvo como objetivo principal realizar una aproximación conceptual de varios autores acerca de la importancia de los Lactobacillus y su papel probiótico en el proceso digestivo y nutricional en cerdos. Para ello, se hizo necesario realizar una profusa búsqueda, selección y procesamiento de las más prominentes fuentes bibliográficas, que tratan sobre este tema. El estudio se llevó a cabo sobre 7 cepas de Lactobacillus y sus diferentes formas de uso en la alimentación de las diferentes categorías porcinas; obteniéndose entre los principales resultados una clara conceptualización de la acción de los Lactobacillus en el sistema inmunológico, genoma y el modo de utilización en bebidas probióticas. A modo de conclusión, se puede mencionar que la totalidad de los autores citados concuerdan que la utilización de Lactobacillus mejora fundamentalmente la sanidad y nutrición porcina, convirtiéndose en la alternativa más viable para remplazar el uso indiscriminado de los antibióticos como promotores de crecimiento.

Palabras Clave: probióticos, antibióticos, proceso digestivo, especies endógenas, categorías porcinas. 


\section{Introducción}

El tracto gastrointestinal de los mamíferos contiene una gran cantidad de microorganismos, microbiota, con los que se establece una relación simbiótica [1]. Su presencia permite la fermentación de sustratos que no podrían ser utilizados por el huésped; aporta moléculas esenciales para las células epiteliales, como vitaminas y ácidos orgánicos; previene el crecimiento de bacterias patógenas, y se ha demostrado que juega un rol fundamental en el desarrollo anatómico, fisiológico e inmunológico del hospedador y que colabora en el mantenimiento de la homeostasis intestinal [2].

Los lactobacilos ocupan una gran variedad de nichos, incluyendo las mucosas de mamíferos $[3,4]$ y distintos tipos de alimentos, entre los que se incluyen productos fermentados lácteos y no lácteos [5-7]. Estas bacterias son comúnmente utilizadas como organismos probióticos en alimentos funcionales, ya que son considerados microorganismos inocuos, principalmente por su uso histórico en la alimentación humana y animal. Por otro lado, se ha demostrado su capacidad para inhibir el crecimiento o invasión de bacterias patógenas y parásitos $[8,9]$ y para modular el sistema inmune [10].

Los experimentos en modelos animales como en lechones en fase de precebo, indican que la microflora intestinal supone una gran cantidad de microorganismos, como las bacterias probióticas con beneficios para el sistema inmune, como lo es la respuesta inmunitaria de la mucosa [11].

La investigación tuvo como objetivo principal el realizar una aproximación conceptual de varios autores acerca de la importancia de los Lactobacillus y su papel probiótico en el proceso digestivo y nutricional en cerdos.

\section{Metodología}

Se realizó una profusa búsqueda, selección y procesamiento de las más prominentes fuentes bibliográficas, que tratan sobre este tema; este estudio, permitió el procesamiento de 67 investigaciones publicadas en idioma inglés, español y portugués, Sede Web (internet), actas de congresos internacionales, revistas indexadas, tesis doctorales. La estrategia de búsqueda asumió como criterios de inclusión que las fuentes consultadas trataran sobre la utilización de Lactobacillus en la alimentación en distintas etapas fisiológicas de cerdos. A partir de herramientas de análisis documental de la información primaria, se asumió como regla el orden cronológico de las publicaciones.

Las principales fuentes consultadas en cada ítem, sobre: Lactobacullus; Lactobacillus plantarum; L. salivarius C65; L. acidophilus; L. casei; L. jensenii; L. rhamnosus L.. Reuteri, fueron las siguientes:

\subsection{En lo que corresponde a lactibacillus}

Land [12]: Lactobacillus sepsis associated with probiotic therapy. Pediatrics; Fradiana [13]: Endocarditis caused by L. jensenii in an immunocompetent patient. 


\subsection{En lo que se refiere a $L$. plantarum}

Pathmakanthan [14]: Beneficial in vitro immunomodulation in cells extracted from inflamed human colon [14]; Stingele [15]: Zwitterionic polysaccharides stimulate T cells with no preferential $\mathrm{V}$ beta usage and promote anergy, resulting in protection against experimental abscess formation; Gámez [16]: Evaluación in vivo de Lactobacillus plantarum como alternativa al uso de antibióticos en lechones; Fredricks [17]: Molecular identifcation of bacteria associated with bacterial vaginosis; Ljungh [18]: Lactobacillus Molecular Biology. From Genomics to Probiotics; Marín [19]: Evaluación de la viabilidad de crecimiento de la cepa nativa. Lactobacillus plantarum LPBM10 y la cepa comercial Lactobacillus casei ATCC 393 en pulpa de uchuva y en solución isotónica de glucosa; Zhan [20]: Production of lactic acid from renewable materials by Rhizopusfungi; González [21]: Aloe vera como sustrato para el crecimiento de Lactobacillus plantarum y Lactobacillus casei. Ciencia y Tecnología Alimentaria; Estela [22]: Producción de ácido láctico por Lactobacillus plantarum L10 en cultivos batch y continuo.

\subsection{En la fundamentación sobre L. salivarius $\mathrm{C} 65$}

Nemcova [23]: In vitro studies of porcine llactobacilli for posible use. Berl; Guerin [24]: Food supplementation with milk fermented by Lactobacillus casei DN - 114001 protects suckling rats from rotavirus associated diarrhea; Iñiguez [25]: Proteincarbohydrate interactions between Lactobacillus salivarius and pig mucins; Pieper [26]: The intestinal microflora of piglets around weaning - with emphasis on Lactobacilli; Fuller [27]: Probiotics in man and animal; Casey [28]: A fvestrain probiotic combination reduces pathogen shedding and alleviates disease signs in pigs chalenged with Salmonella enterica serovar Typhimurium. Appl. Environ. Microbiol; Yeo [29]: Effect of feeding diets containingan antibiotic, a probiotic, or yucca extract on growth and intestinal urease activity in broiler chicks. Poult.

\subsection{En la argumentación sobre $L$. acidophilus}

Yeo [29]: Effect of feeding diets containingan antibiotic, a probiotic, or yucca extract on growth and intestinal urease activity in broiler chicks. Poult.

\subsection{En lo que corresponde a $L$. casei}

Mejía [30]: Evaluación de dos probióticos sobre parámetros productivos en lechones lactantes; Holzapfel [31]: Taxonomy and important features of probiotic microorganisms in food and nutrition; Spanhaak [32]: The effect of consumption of milk fermented by Lactobacillus casei strain Shirota on the intestinal microflora and immune parameters in humans [32]; Matsuzaki [33]: Immunomodulation by treatment with Lactobacillus casei strain Shirota; Reid [34]: Minireview: The scientific basis for probiotic strains of Lactobacillus. 


\subsection{En la fundamentación sobre $L$. rhamnosus}

Bomba [35]: Potentation of the effectiveness of Lactobacillus casei in the prevention of $E$. coli induced diarrea in conventional and gnotobiotic pigs; Alander [36]: Recovery of Lactobacillus rhamnosus GG from human colonic biopsies; Altech [37]: BIO-MOS in the poultry industry. Biotech in the feed industry. Supplement to the Proc. Alltech's Biotech; Avila [38]: A comparative study of the efficiency of a probiotic and anti-K99 and antiA14 vaccines in the control of diarrhea in calves in Brazil; Alltech [39]: BIO-MOS in the poultry industry. Biotech in the feed industry. Supplement to the Proc; Boucourt [40]: Efecto de la actividad probiótica de Lactobacillus rhamnosus en indicadores fisiológicos de lechones.

\subsection{En lo que se refiere a Lactobacillus jensenii}

Biricik [41]: The effect of Saccharomyces cerevisiae on in vitro rumen digestibilities of dry matter, organic matter and neutral detergent fibre of different forage: concenrate ratios in diets; Shimazu [42]: Immunobiotic Lactobacillus jensenii elicit anti-inflammatory activity in porcine intestinal epithelial cells by modulating negative regulators of the toll-like receptorsignaling pathway; Villena [43]: Immunobiotic Lactobacillus jensenii modulates toll-like receptor 4-induced inflammatory response via negative regulation in porcine antigen presenting cells.

\subsection{En la argumentación sobre Lactobacillus Reuteri}

Oh [44]: Diversification of the gut symbiont Lactobacillus reuteri as a result of hostdriven evolution; Chang [45]: Selection of a potential probiotic Lactobacillus strain and subsequent in vivo studies; Hou [46]: Complete genome sequence of Lactobacillus reuteri 15007, a probiotic strain isolated from healthy piglet; Hou [47]: Study and use of the probiotic Lactobacillus reuteri in pigs; Huang [48]: Effects of Lactobacillus on the performance, diarrhea incidence, VFA concentration and gastrointestinal microbial flora of weaning pigs; Chang [49]: Selection of a potential probiotic Lactobacillus strain and subsequent in vivo studies; Yu [50]: The effects of probiotic Lactobacillus reuteri Pg4 strain on intestinal characteristics and performance in broilers; Seo [51]: Bile tolerant Lactobacillus reuteri isolated from pig feces inhibits enteric bacterial pathogens and porcine rotavirus; Urbanska [52]: The efficacy of Lactobacillus reuteri DSM 17938 in infants and children: A review of the current evidence; Lee [53]: Isolation, characterization, and evaluation of wild isolates of Lactobacillus reuteri from pig feces; Hou [54]: Complete genome sequence of Lactobacillus reuteri 15007 , a probiotic strain isolated from healthy piglet; Heavens [55]: Genome sequence of the vertebrate gut symbiont Lactobacillus reuteri ATCC 53608; Boucourt [40]: Efecto de la actividad probiótica de Lactobacillus rhamnosus en indicadores fisiológicos de lechones; Rosander [56]: Removal of antibiotic resistance genecarrying plasmids from Lactobacillus reuteri ATCC 55730 and characterization of the resulting daughter strain, Lactobacillus reuteri DSM 17938. App/ Environ Microbiol; van de Guchthe [57]: Stress responses in lactic acid bacteria; Liu 
[58]: Fermentation conditions influence the fatty acid composition of the membranes of Lactobacillus reuteri 15007 and its survival following freeze-drying; Lebeer [59]: Genes and molecules of lactobacilli supporting probiotic action. Microbiol Mol Biol Rev; Bian [60]: An in vitro antimicrobial and safety study of Lactobacillus reuteri DPC16 for validation of probiotic concept. Master thesis; Morita [61]: Comparative genome analysis of Lactobacillus reuteri and Lactobacillus fermentum reveal a genomic island for reuterin and cobalamin production; Gänzle [62]: Characterization of reutericyclin produced by Lactobacillus reuteri LTH2584. App/ Environ Microbiol; Mukai [63]: Inhibition of binding of Helicobacter pylori to the glycolipid receptors by probiotic Lactobacillus reuteri. FEMS Immunol Med Microbiol; Taranto [64]: Lactobacillus reuteri CRL1098 produces cobalamin; Wang [65]: Free radical scavenging activity of Lactobacillus fermentum in vitro and its antioxidative effect on growingfinishing pigs; Hoffmann [66]: LactobacilIus reuteri 100-23 transiently activates intestinal epithelial cells of mice that have a complex microbiota during early stages of colonization; Dicksved [67]: Lactobacillus reuteri maintains a functional mucosal barrier during DSS treatment despite mucus layer dysfunction.

\section{Resultados y Discusión}

\subsection{Lactobacillus}

En general, los lactobacilos son considerados microorganismos GRAS (generally regarded as safe) por la FDA (Administración de Alimentos y Drogas de Estados Unidos de América), y la mayor parte de ellos, como L. kefiri, se encuentran incluidos en la lista del QPS (qualified presumption of safety) de la Unión Europea (EFSA Panel on Biological Hazards (BIOHAZ) 2012). Esto se debe a dos motivos principales: El primero es su uso histórico y seguro en productos fermentados y, el segundo es que forman parte de la microbiota del tracto gastrointestinal de los monogastricos Sin embargo, ciertas especies de lactobacilos han sido identificadas en casos de sepsis, endocarditis o bacteriemias, mayormente en pacientes con enfermedades crónicas severas [12, 13].

\subsection{Lactobacillus plantarum}

En lo que se refiere a bacterias acido lácticas el L. plantarum es capaz de promover la síntesis y secreción de citocina antiinflamatoria IL-10 en los macrófagos y células T que proceden del colon inflamado [14].

\subsubsection{Lactobacillus plantarun y su acción en el sistema inmunológico}

La flora endógena intestinal sintetiza los polisacáridos capsulares zwitteriónicos (PSZ), los cuales, emplean el sistema de presentación del complejo mayor de histocompatibilidad II (MHC) para activar las células T mediante su reconocimiento por las 
proteínas receptoras alfa/beta de dichas células T. Así, por ejemplo, células T CD4+ humanas estimuladas por estas moléculas in vitro y transferidas a ratas in vivo protegen contra abscesos intraabdominales provocados con un estímulo bacteriano viable [15].

Se realizó un estudio de in vivo sobre la sustitución o complemento al uso de antibióticos mediante la utilización de inóculos probióticos de L. plantarum, junto con la ración de iniciación libre de antibióticos en fase de precebo, y así lograr un mejor control de las infecciones que causan diarrea. Además, se evaluó su efecto en los pesos vivos fnales, niveles de inmunoglobulina $A$, colesterol total y nitrógeno ureico, eficacia de la colonización por microscopía electrónica de alta resolución y análisis microbiológicos de muestras histológicas del intestino grueso y materia fecal en los grupos seleccionados [16].

\subsubsection{Cepas de L. plantarun}

Los resultados de la identificación molecular indicaron que las secuencias parciales del gen que codifica para el RNA ribosomal $16 \mathrm{~S}$ de las cepas de $L$. plantarum $1 \mathrm{H} 1$ y $L$. plantarum $1 \mathrm{H} 2$ presentaron una similitud del 97 al 99\%, y del 95\% respectivamente con las secuenciasdel gen del RNA ribosomal $16 \mathrm{~S}$ de los linajes $L$. plantarum, L. pentosus, $L$. paraplantarum y $L$. plantarum subsp. argentorantensis, incluyendo los linajes tipo cuyos No. de acceso Genbakson D79210, D79211, AJ306297 y AJ 640078. También presentaron entre el 99 y $100 \%$ desimilaridad con las secuencias del gen RNA ribosomal $16 S$ de aislados de Lactobacillus spp., presentes en las bases de datos consultadas Genbak y RDP [16].

\subsubsection{Ensayos de laboratorio con L. plantarum.}

\section{Elaboración de inocluos}

Gamez et al. [16] manifiestan que para el proceso de elaboración de los inóculos, la fermentación se interrumpió al final de la fase logarítmica, es decir, a las doce horas, y posteriormente, se evaluó la viabilidad (UFC/ml) y $\mathrm{pH}$ a temperatura ambiente $\left(29,5^{\circ} \mathrm{C}\right)$ y refrigeración $\left(4^{\circ} \mathrm{C}\right)$ en volúmenes $250 \mathrm{ml}$ y dos litros en períodos de: $12 \mathrm{hr}, 7,12$ y 21 días para cada volumen.

Gamez et al. [16] reportan que al evaluar la viabilidad de las dos bacterias seleccionadas por un período de 7 días, presentaron una alta viabilidad tanto a temperatura de refrigeración como ambiental, con valores de 2,0×1014 con pH de 3,83 y 2,0×1012 UFC/ml con pH de 3,57 respectivamente para L. plantarum $1 \mathrm{H} 1 \mathrm{y}$, de 2,0×1014 con pH de 3,76 y 4,0×1012 UFC/ml con $\mathrm{pH}$ de 3,57 respectivamente para $L$. plantarum $1 \mathrm{H} 2$.

Según Gamez et al. [16] reportan que la viabilidad para un período de 12 días mostró una diferencia marcada, por cuanto $L$. plantarum $1 \mathrm{H} 1$ presentó valores superiores de $6,0 \times 1013 \mathrm{UFC} / \mathrm{ml}$ en refrigeración, mientras que a temperatura ambiente $\left(25^{\circ} \mathrm{C}\right)$ fue de 1,0×109 UFC/ml. En cuanto al pH, presentaron valores de 3,51 y 3,43. Para el caso de L. plantarum 1 H2 indicó ser de igual manera, más viable en refrigeración (1,0×1010 
UFC/ml) que a temperatura ambiental $(9,0 \times 109$ UFC/ml). Para el caso del pH, presentó valores de 3,60 y 3,46 .

Según Gamez et al. [16] manifiestan que los resultados que dieron el primer parámetro para la producción de los inóculos, ya que permitiría mantener en refrigeración hasta por 12 días los inóculos de L. plantarum 1 H2 manteniendo una viabilidad alta y un $\mathrm{pH}$ cercano al de los lechones destetos. Por el contrario, L. plantarum $1 \mathrm{H} 1$ podría ser almacenado por más tiempo en refrigeración dada su alta viabilidad.

Gamez et al. [16] reportan que el análisis flogenético confrmó la similaridad de $L$. plantarum $1 \mathrm{H} 1$ y L. plantarum $1 \mathrm{H} 2$ en un agrupamiento soportado con alto valor de bootstrap (100\%), relacionado con los linajestipo de las especies de $L$. plantarum, $L$. pentosus, L. paraplantarum y L. plantarumsubsp. Argentorantensis [16].

Marin et al. [19] dan a conocer que la supervivencia de las cepas L. plantarum LPBM10 y L. casei ATCC 393, en sustratos de pulpa de uchuva y solución de glucosa (14\% $\mathrm{p} / \mathrm{p}$ ), y almacenamiento a $4^{\circ} \mathrm{C}$ durante 15 días. A los 15 días obtuvieron viabilidades en pulpa y solución de glucosa, para $L$. plantarum de 3,23 $\pm 3,35 \times 109$ y 1,64 $\pm 1,57 \times 109$ $\mathrm{UFC} / \mathrm{ml}$, y para $L$. casei de $5,40 \pm 2,36 \times 108$ y $7,34 \pm 7,88 \times 108 \mathrm{UFC} / \mathrm{ml}$, respectivamente. Los autores concluyeron que con niveles superiores a 106, son unos de los criterios considerados para defnir un alimento con probióticos. Estos resultados coinciden con los encontrados para un período de 12 días [21] a temperatura de refrigeración y confrman que $L$. plantarum $1 \mathrm{H} 1$ y especialmente $L$. plantarum $1 \mathrm{H} 2$ son viables a esta temperatura [22].

\section{Genoma del L. plantarun}

La versatilidad de L. plantarum se debe al tamaño de su genoma, el cual es 50\% más grande que la mayoría de las BAL y tiene una gran capacidad metabólica, que le permite utilizar una gran variedad de fuentes de carbono; propiedad que resulta de un gran número de genes involucrados en el transporte y utilización de azúcar, y un metabolismo versátil del piruvato, el cual tiene el potencial de producir D y L-lactato, formato, acetato, etanol, acetoína y 2,3-butanediol [17, 18]. Las BAL tienen la capacidad de crecer en una variedad de sustratos $[19,20]$.

\subsection{Lactobacillus salivarius $\mathrm{C} 65$}

Lactobacillus salivarius se encuentra normalmente en la población microbiana que reside en el tracto digestivo de todos los animales de interés zootécnico, como los cerdos [23]. Las cepas de L. salivarius se adhieren a los carbohidratos de la mucosa intestinal a través de proteínas denominadas lectinas, presentes en la superfcie de las bacterias. Esta característica contribuye a la protección de la mucosa y a la estimulación de la actividad inmune $[24,25]$. 


\subsubsection{Lactobacillus salivarius parte de la microflora intestinal en cerdos}

Los L. salivarius, son los lactobacilos más abundantes de la comunidad microbiana del íleon de cerdos durante el período del destete [26]. Si se provee a los animales de cepas autóctonas del tracto gastrointestinal mediante el uso de probióticos desde las primeras horas de su nacimiento, estas bacterias colonizarán la mucosa intestinal y la protegerán de forma natural contra el crecimiento de otros microorganismos, especialmente de aquellos que son dañinos o indeseables [27].

Casey et al. [28] informan que al suministrar una mezcla de cepas de L. murinus, L. salivarius, L. pentosus y Pediococcus pentosaceous a lechones destetados produjo menor incidencia, severidad y duración de diarreas, cuando se suministró por vía oral una cepa de Salmonella typhimurium [28].

\subsubsection{Biopreparados con L. salivarius}

El biopreparado de L. salivarius influyó en el incrementa del peso vivo de los cerditos. Se conoce que los microorganismos que residen en el tracto gastrointestinal interactúan con el animal hospedero. Esta microbiota varía con la especie animal, el sitio del sistema digestivo donde se aloja, la edad, la dieta que consume y el ambiente. Los animales saludables mantienen una población microbiana balanceada, lo que se corresponde con el estado eubiósico del ecosistema gastrointestinal. Esta condición se relaciona estrechamente con la productividad y salud de los animales [29].

\subsection{Lactobacillus acidophilus}

\subsubsection{Biopreparados con L. acidophilus}

Yeo y Kim [29] manifiestan que el biopreparado con L. acidophilus se suministró a crías porcinas lactantes directamente por vía oral o en el pienso, lo que mejoró el incremento de peso y la ganancia media diaria. Además, la incidencia de diarreas disminuyó, y no se presentaron muertes por trastornos digestivos. Estos resultados se pudieran atribuir a la función que desempeñan las bacterias ácido lácticas en el tracto gastrointestinal de los cerditos jóvenes, pues el ácido láctico derivado de los lactobacilos provee la acidez necesaria para los procesos digestivos. Se conoce que hasta la tercera o cuarta semana de vida no se intensifica la secreción de ácido clorhídrico. La acidez del estómago en los cerditos contribuye al control de gérmenes patógenos y a la acidez óptima para la actividad de la pepsina, que debe estar entre pH 2 y 4 [29].

\subsection{Lactobacillus casei}

\subsubsection{Probióticos con L. casei}

Esta bacteria es un microorganismo reconocido como seguro y se encuentra dentro de las especies más comunes que se emplean en las preparaciones probióticos 
[30]. Lactobacillus casei, cepa Shirota ha sido ampliamente estudiada y se describió como agente probiótico al ser capaz de colonizar el epitelio intestinal [31] estimular la respuesta inmune y prevenir infecciones enterobacterianas [32]. En Tokyo, esta cepa se utiliza para la fabricación del probiótico comercial [33] para prevenir la adhesión de E. coli O8:K88 en el tracto gastrointestinal de cerdos gnotobióticos y convencionales. Varios autores observaron que la administración del Lactobacillus con la maltodextrina disminuyó significativamente el número de $E$. coli que colonizaron el yeyuno de los cerdos convencionales en comparación con el grupo control [34].

\subsection{Lactobacillus rhamnosus}

\subsubsection{Probióticos con L. rhamnosus}

Entre las especies de Lactobacillus usados se encuentra el L. rhamnosus. Con la utilización de los probióticos, se han logrado buenos resultados en varios países [3538]. Sin embargo, en Cuba no hay experiencias en su obtención y uso como aditivo alimentario, a pesar de que estos pueden obtenerse con recursos nacionales y de bajo costo. Por esto, el objetivo de este estudio fue determinar la actividad probiótica de $L$. rhamnosus en indicadores fisiológicos de lechones [39].

Boucourt et al. [40] reportan en su ensayo con L. rhamnosus, procedente del cepario del Instituto Cubano de Investigación de los Derivados de la Caña de Azúcar (ICIDCA), se suministró en una dosis de $2 \times 109$ ufc/animal, por vía oral el primer, tercer y decimocuarto día de edad, para que coincidiera con las manipulaciones zootécnicas de los cerditos. A partir de este momento, se mezcló con el pienso de inicio por atomización y homogenización manual, a razón de 108 ufc/g (un promedio de 1010 /cerdito/d), hasta el final del experimento.

Boucourt et al. [40] manifiestan que los lechones, de un cruce comercial YorkshireLandrace x L35 y descendientes de cerdas de primer parto, se destetaron a los $42 \mathrm{~d}$ de edad. Las camadas de cada tratamiento se ubicaron distantes unas de otras (con un bóxer intermedio o a ambos lados del pasillo), para evitar la autoinoculación entre ellas. Tanto las lactantes como los cerditos de los dos tratamientos, se sometieron a similares condiciones de manejo. Para medir el efecto probiótico, se determinaron los indicadores morfométricos del intestino delgado al destete y la microbiología del ciego, además de los niveles de colesterol y de urea en el suero, a los 42 y 63 días de edad. En este último período, también se midieron los indicadores morfométricos del timo y del corazón. Los medios de cultivo utilizados fueron: agar extracto de malta para determinar las levaduras y hongos, agar violeta rojo bilis para los coliformes y el agarogosa para la determinación de los lactobacilos, así como el agar nutriente en los aerobios totales [40]. 


\subsection{Lactobacillus jensenii}

\subsubsection{Lactobacillus jensenii y su acción en el sistema inmunológico}

Shimazu et al. [42] reportan que el Lacttobacillus jensenii atenúa la expresión de citoquinas proinflamatorias y quimiocinas activadas por ETEC o lipopolisacárido (LPS) en una línea celular de epiteliocitos intestinales porcinos (PIE) [40]. Lacttobacillus jensenii TL2937 atenúa las respuestas proinflamatorias en células PIE regulando a la baja el factor nuclear dependiente KB (NF-KB) dependiente de Tollliker (TLR) - 4 y mitógeno activada por la proteína quinasa (MAPK). Además, se ha demostrado que la estimulación L. jensenii TL2937 de las células PIE da como resultado una regulación positiva de tres reguladores negativos de los TLR, la enzima de edición de ubiquitina A20, la proteína codificada por el linfoma Bcell 3 (Bcl-3) y la proteína quinasa activada por el mitógeno (MPK-1) y que estos efectos son parcialmente dependientes de la activación de TLR2 [41]. Más recientemente, han evaluado el efecto del TL2937 en células presentadoras de antígeno (APC) de porcino Parches de Peyer (PP) y han encontrado que la exposición directa de Las APC porcinas a L. jensenii TL2937 en ausencia de señales inflamatorias activaron las APC CD172a + y causaron que se vuelvan fenotípicamente y funcionalmente maduros, y para mostrar propiedades tolerogénicas.

\subsection{Lactobacillus Reuteri}

Lactobacillus reuteri es una de las especies dominantes en el TGI de vertebrados como humanos, ratas, cerdos y pollos [43]. Es una de las especies probióticas mejor documentadas y se ha utilizado ampliamente como probiótico en humanos y animales [44].

En los últimos años, se han usado numerosas cepas probióticas en la producción porcina. La aplicación de probióticos proporciona una estrategia alternativa potencial al uso de antibióticos [45].

\subsubsection{Lactobacillus reuteri parte de la microflora intestinal}

Lactobacillus reuteri es una bacteria heterofermentativa, y se considera una de las pocas especies de Lactobacillus autóctonas verdaderas en humanos y animales. Muchos investigadores ya han seleccionado algunas cepas específicas de $L$. reuteri aisladas de heces humanas, leche materna, vagina humana, cavidad oral humana, cobayas, ratas, cerdos, pollos de engorde y masa fermentada. Ahora hay una creciente evidencia para demostrar que las cepas seleccionadas de $L$. reuteri tienen características probióticas y pueden proporcionar beneficios para la salud a sus huéspedes [46]. 


\subsubsection{Cepas probioticas de $L$. reuteri}

Las bacterias probióticas se encuentran con varias tensiones después de la ingestión por parte del huésped, incluida la exposición a un pH bajo en el estómago y el contacto con la bilis en el intestino delgado. Lactobacillus reuteri 15007, inicialmente conocido como L. fermentum 15007 , se seleccionó entre más de 7000 colonias nativas de Lactobacillus según criterios que incluyen resistencia al calor, $\mathrm{pH}$ bajo, cobre y sales biliares, así como estabilidad de almacenamiento y antagonismo a agentes patógenos [47]. Otras cepas de $L$. reuteri también muestran resistencia a $\mathrm{pH}$ bajo y bilisSales [47-49].

Lactobacillus reuteri tiene el registro de evaluación de seguridad más extenso de todas las cepas probióticas. Varios estudios realizados tanto in vivo como in vitro indican que $L$. reutei es seguro para el consumo humano, incluso en grandes cantidades [50, 51]. Sin embargo, como es el caso de todas las demás especies de LAB, se pueden encontrar plásmidos en algunas cepas de L. reuteri [52-54], y se ha demostrado que algunos de estos plásmidos codifican genes de resistencia a antibióticos [55].

Según la Autoridad Europea de Seguridad Alimentaria, los probióticos no deben contener rasgos conocidos de resistencia a los antibióticos. Lactobacillus reuteri ATCC 55730 es una cepa probiótica disponible comercialmente que se ha encontrado que posee rasgos de resistencia potencialmente transferibles para la tetraciclina y la lincomicina. Por lo tanto, ha sido reemplazado por L. reuteri DSM 17938, una cepa donde los dos plásmidos de resistencia se eliminaron sin perder ninguna característica probiótica [56].

Las cepas probióticas deben ser capaces de resistir cualquier condición adversa encontrada durante la producción industrial para poder sobrevivir [57]. Lactobacillus reuteri es sensible al calor y, por lo tanto, el secado por congelación se usa comúnmente para mantener la estabilidad de $L$. reuteri. Someter a $L$. reuteri a una temperatura de fermentación más alta $\left(47^{\circ} \mathrm{C}\right)$ o un $\mathrm{pH}$ neutro $(\mathrm{pH} 6,7)$ se ha demostrado que aumenta la supervivencia de $L$. reuteri durante el posterior secado por congelación [58].

\subsubsection{Lactobacillus reuteri y su función antimicrobiana}

Se ha informado que $L$. reuteri produce una variedad de sustancias antimicrobianas como el ácido láctico, el peróxido de hidrógeno [59], la reuterina [60, 61] y la reutericiclina [62], que tienen efectos beneficiosos para el organismo huésped. Se ha demostrado que las cepas de $L$. reuteri inhiben el crecimiento de muchos patógenos entéricos, como E. coli, S. Typhimurium, Staphylococcus epidermidis, S. aureus, $H$. pylori y rotavirus $[47,49,63]$. Además, $L$. reuteri puede producir vitamina B12 $[61,64]$, y tiene la capacidad de sintetizar de nuevo la L-lisina y el ácido fólico a partir de un modelo de simulación por computadora [45]. 


\subsubsection{Lactobacillus reuteri y su acción en el sistema inmunológico}

Lactobacillus reuteri exhibió capacidad de eliminación de radicales libres in vitro [65], y codificó varias enzimas antioxidantes [45]. Los estudios en animales y humanos han demostrado que la administración oral de $L$. reuteri redujo la incidencia y la gravedad de la diarrea, disminución del dolor visceral, prevención de la enterocolitis cólicos y necrótica, mantenimiento de la barrera mucosa funcional, e induce a la colonización e inmunomodulación $[66,67]$. Lactobacillus reuteri en lechones neonatales se puede usar para apoyar el desarrollo de una microbiota estable, para estimular el sistema inmunológico y para prevenir enfermedades diarreicas. Durante los períodos de destete y posterior al destete, $L$. reuteri se utiliza en cerdos para modular la microbiota gastrointestinal y para prevenir la diarrea posterior al destete y estimular crecimiento. Lactobacillus reuteri 15007 desempeña un papel positivo en el desarrollo intestinal en lechones neonatales Mediante la modulación de la composición microbiana e intestinal [46]. El desarrollo, la electroforesis en gel de gradiente desnaturalizante (DGGE) reveló que $L$. reuteri 15007 afectó a las comunidades microbiológicas colónicas en el día 14 y, en particular, redujo el número de Clostridium spp. En los cerdos destetados, la administración de L. reuteri BSA131 redujo el número de bacterias en las heces [47].

\section{Conclusiones}

Luego de haber realizado la búsqueda, selección y procesamiento de la información de 67 autores, citados sobre los Lactobacillus y su papel probiótico en el proceso nutricional y digestivo en cerdos, se llegaron a conceptualizar siete cepas de Lactobacillus y sus aplicaciones en la alimentación en esta especie. En cada una de las investigaciones revisadas, se pudo evidenciar que el accionar de los probióticos mejora el sistema inmunológico en correlación estrecha la calidad de vida del animal y, de esta manera, se podrá cumplir con los objetivos de producción de una manera eficaz y eficiente.

\section{Agradecimientos}

La realización de esta investigación está dedicada aquellas personas que de alguna manera fueron participes de la misma. Nuestros sinceros agradecimientos están dirigidos a la Escuela Superior Politécnica de Chimborazo - Facultad de Ciencias Pecuarias precursoras de la investigación científica.

\section{Conflicto de Intereses}

No existen conflicto de intereses.

\section{References}

[1] Kelly D, Mulder IE. Microbiome and immunological interactions. Nutrition Reviews. 2012;70(Suppl 1):1830. 
[2] Gu S, Chen D, Zhang J-N et al. Bacterial community mapping of the mouse gastrointestinal tract. PloS One. 2013;8(10):749-57.

[3] Sekirov I, Russell S, Antunes CM, Finlay BB. Gut microbiota in health and disease. Physiological Reviews. 2010;90:859-904.

[4] Martín R, Sánchez B, Suárez JE, Urdaci MC. Characterization of the adherence properties of human Lactobacilli strains to be used as vaginal probiotics. FEMS Microbiology Letters. 2012;328(2):166-73.

[5] Garrote G, Abraham A, de Antoni G. Chemical and microbiological characterisation of kefir grains. Journal of Dairy Research. 2001;68(4):639-52.

[6] Bernardeau M, Vernoux JP, Henri-Dubernet S, Guéguen M. Safety assessment of dairy microorganisms: The Lactobacillus genus. International Journal of Food Microbiology. 2008;126(3):278-85.

[7] Dušková M, Šedo O, Kšicová K, Zdráhal Z, Karpíšková R. Identification of lactobacilli isolated from food by genotypic methods and MALDI-TOF MS. International Journal of Food Microbiology. 2012;159(2):107_ 14.

[8] Servin AL. Antagonistic activities of lactobacilli and bifidobacteria against microbial pathogens. FEMS Microbiology Reviews. 2004;28(4):405-40.

[9] Humen M, de Antoni G, Benyacoub J et al. Lactobacillus johnsonii La1 antagonizes Giardia intestinalis in vivo. Infection and Immunity. 2005;73(2):1265-1269. Corthésy B, Gaskins H, Mercenier A. 2007. Crosstalk between probiotic bacteria and the host immune system. The Journal of Nutrition. 2007;781-790.

[10] Yamamoto M, Sato S, Hemmi H et al. Essential role for TIRAP in activation of the signalling cascade shared by TLR2 and TLR4. Natur. 2002;420:324-329.

[11] Land MH, Rouster-Stevens K, Woods CR, Cannon ML, Cnota J, Shetty AK. Lactobacillus sepsis associated with probiotic therapy. Pediatrics. 2005;115(1):178-81.

[12] Fradiani PA, Petrucca A, Ascenzioni $F$ et al. Endocarditis caused by Lactobacillus jensenii in an immunocompetent patient. Journal of Medical Microbiology. 2010;59(5):607-9.

[13] Pathmakanthan S, Li CK, Cowie J, Hawkey CJ. Lactobacillus plantarum 299: beneficial in vitro immunomodulation in cells extracted from inflamed human colon. J Gastroenterol Hepatol. 2004;19:166173.

[14] Stingele F, Corthesy B, Kusy N, Porcelli SA, Kasper DL, Tzianabos AO. Zwitterionic polysaccharides stimulate $T$ cells with no preferential $\mathrm{V}$ beta usage and promote anergy, resulting in protection against experimental abscess formation. J Immunol. 2004;172:1483-1490.

[15] Gámez HJ, Ramírez C, Martínez J. 2013. Evaluación in vivo de Lactobacillus plantarum como alternativa al uso de antibióticos en lechones. Revista MVZ Córdoba. 2013;18(3):3648-3657.

[16] Fredricks DN, Fiedler TL, Marrazzo JM. Molecular identifcation of bacteria associated with bacterial vaginosis. N Engl J Med. 2005; 353:1899-1911.

[17] Ljungh A, Wadstrom T. Editores. Lactobacillus molecular biology. From genomics to probiotics. Norfolk: Caister Academic Press; 2009.

[18] Marín Z, Cortés M, Montoya O. Evaluación de la viabilidad de crecimiento de la cepa nativa Lactobacillus plantarum LPBM10 y la cepa comercial Lactobacillus casei ATCC 393 en pulpa de uchuva y en solución isotónica de glucosa. Rev VITAE. 2009; 16(2):210-217.

[19] Zhan YZ, Bon J, Joan MK. Production of lactic acid from renewable materials by Rhizopus fungi. Chem Eng Process. 2006; 46:361-374.

[20] González BA, Domínguez R, Alcocer BR. Aloe vera como sustrato para el crecimiento de Lactobacillus plantarum y L. casei. Ciencia y Tecnología Alimentaria. 2008;6(2):152-157.

[21] Estela W, Rychtera M, Melzoch K, Quillama E, Egoavil E. Producción de ácido láctico por Lactobacillus plantarum L10 en cultivos batch ycontinuo. Rev Perú Biol. 2007; 14(2):271-276.

[22] Nemcova R, Laukova A, Gancarcikova S, Kastel R. In vitro studies of porcine llactobacilli for posible use. Berl. Munch. Tieraztl. Wschr. 1997;110:413.

[23] Guerin C, Meslin J, Chambard A et al. 2001. Food supplementation with milk fermented by Lactobacillus casei DN - 114001 protects suckling rats from rotavirus associated diarrhea. J. Nutr. 2001;131:111

[24] Iñiguez C, Jiménez R, Vázquez L, Ramos G, Acedo E. Proteincarbohydrate interactions between Lactobacillus salivarius and pig mucins. J. Nutr. 2011;132:110

[25] Pieper R, Janczyk P, Schumann R, Souffrant WB. The intestinal microflora of piglets around weaning With emphasis on lactobacilli. Arch. Zootech. 2006;9:29.

[26] Fuller R. Probiotics in man and animal. J. Appl. Bacteriol. 1989;66:365.

[27] Casey P, Gardiner G, Casey G et al. A fvestrain probiotic combination reduces pathogen shedding and alleviates disease signs in pigs chalenged with Salmonella enterica serovar Typhimurium. Appl. Environ. Microbiol. 2007;73:1858.

[28] Yeo J, Kim K. Effect of feeding diets containingan antibiotic, a probiotic, or yucca extract on growth and intestinal urease activity in broiler chicks. Poult. Sci. 1997;76:381 
[29] Mejía W, Rubio J, Calatayud D, Rodríguez A, Quintero, A. Evaluación de dos probióticos sobre parámetros productivos en lechones lactantes. Zootécnia Trop. 2007;25:301.

[30] Holzapfel WH, Haberer P, Geisen R, Björkroth J, Schillinger U. Taxonomy and important features of probiotic microorganisms in food and nutrition. American Journal of Clinical Nutrition. 2001; 73(supplement):365-373.

[31] Spanhaak S, Havenaar R, Schaafsma G. The effect of consumption of milk fermented by Lactobacillus casei strain Shirota on the intestinal microflora and immune parameters in humans. European Journal of Clinical Nutrition. 1998;52:899-907.

[32] Matsuzaki T. Immunomodulation by treatment with Lactobacillus casei strain Shirota. International Journal of Food Microbiology. 1998;41:133-140.

[33] Reid G. Minireview: The scientific basis for probiotic strains of Lactobacillus. Applied Environmental Microbiology. 1999;65:3763-3766.

[34] Bomba A, Nemcová R, Gancarčíková S, Herich R, Kaštel R. Mechanisms in the pathogenesis of enteric diseases 2. (P.S. Paul, y D.H. Francis, editores). New York: Kluwer Academic/Plenum Publishers; 1999. New York Potentation of the effectiveness of Lactobacillus casei in the prevention of $E$. coli induced diarrea in conventional and gnotobiotic pigs. p. 185-190

[35] Alander M, Korpela R, Saxelin M, Salmela VT, Mattila-Sandholm T, von WA. Recovery of Lactobacillus rhamnosus GG from human colonic biopsies. Lett. Appl. Microbiol. 1997;24:361.

[36] Alltech. BIO-MOS in the poultry industry. Biotech. in the feed industry. Supplement to the Proc. Alltech's Biotech. Feed Industry. Lyons TP, editor. Nicholasville; 1995.

[37] Avila F, Parlillo A, Schocken R, Lucas F, Orgaz A, Quintana J. A comparative study of the efficiency of a probiotic and anti-K99 and antiA14 vaccines in the control of diarrhea in calves in Brazil. Rev. Elev. Med. Vet. Pays. Trop. 1995;48:239.

[38] Alltech. BIO-MOS in the poultry industry. Biotech. In the feed industry. Supplement to the Proc. Alltech's Biotech. Feed lindustry. Lyons TP, editor. Nicholasville; 1996.

[39] Boucourt R, Savón L, Díaz J et al. Efecto de la actividad probiótica de Lactobacillus rhamnosus en indicadores fisiológicos de lechones. Revista Cubana de Ciencia Agrícola. 2004;38(4):411-416.

[40] Biricik H, Türkmen II. The effect of Saccharomyces cerevisiae on in vitro rumen digestibilities of dry matter, organic matter and neutral detergent fibre of different forage: Concenrate ratios in diets. J. Fac. Vet. Med. 2001:20:29.

[41] Shimazu T, Villena J, Tohno M et al. Immunobiotic Lactobacillus jensenii elicit anti-inflammatory activity in porcine intestinal epithelial cells by modulating negative regulators of the toll-like receptor signaling pathway. Infect Immun. 2012;80:276-288.

[42] Villena J, Suzuki R, Fujie H et al. Immunobiotic Lactobacillus jensenii modulates toll-like receptor 4 induced inflammatory response via negative regulation in porcine antigen presenting cells. Clin Vaccine Immunol. 2012;19:1038-1053.

[43] Oh PL, Benson AK, Peterson DA et al. Diversification of the gut symbiont Lactobacillus reuteri as a result of host-driven evolution. ISME J. 2010; 4:377-87.

[44] Chang YH, Kim JK, Kim HJ, Kim WY, Kim YB, Park YH. Selection of a potential probiotic Lactobacillus strain and subsequent in vivo studies. Antonie Van Leeuwenhoek. 2001;80:193-9.

[45] Hou C, Wang Q, Zeng $X$ et al. Complete genome sequence of Lactobacillus reuteri I5007, a probiotic strain isolated from healthy piglet. J Biotechnol. 2014;179:63-4.

[46] Hou C, Zeng X, Yang F, Liu H, Qiao S. (2015). Study and use of the probiotic Lactobacillus reuteri in pigs: A review. Journal of animal science and biotechnology. 2015;6(1):14.

[47] Huang CH, Qiao SY, Li DF, Piao XS, Ren JP. Effects of Lactobacillus on the performance, diarrhea incidence, VFA concentration and gastrointestinal microbial flora of weaning pigs. Asian-Aust J Anim Sci. 2004;17:401-9.

[48] Chang YH, Kim JK, Kim HJ, Kim WY, Kim YB, Park YH. Selection of a potential probiotic Lactobacillus strain and subsequent in vivo studies. Antonie Van Leeuwenhoek. 2001;80:193-9.

[49] Yu B, Liu JR, Chiou MY, Hsu YR, Chiou PWS. The effects of probiotic Lactobacillus reuteri Pg4 strain on intestinal characteristics and performance in broilers. Asian-Aust J Anim Sci. 2007;20:1243-51.

[50] Seo BJ, Mun MR, Kumar JR et al. Bile tolerant Lactobacillus reuteri isolated from pig feces inhibits enteric bacterial pathogens and porcine rotavirus. Vet Res Commun. 2010; 34:323-33.

[51] Urbanska M, Szajewska H. The efficacy of Lactobacillus reuteri DSM 17938 in infants and children: A review of the current evidence. Eur J Pediatr. 2014;173:1327-37.

[52] Lee DY, Seo YS, Rayamajhi N, Kang ML, Lee SI, Yoo HS. Isolation, characterization, and evaluation of wild isolates of Lactobacillus reuteri from pig feces. J Microbiol. 2009;47:663-72.

[53] Hou C, Wang Q, Zeng $X$ et al. Complete genome sequence of Lactobacillus reuteri I5007, a probiotic strain isolated from healthy piglet. J Biotechnol. 2014;179:63-4. 
[54] Heavens D, Tailford LE, Crossman L et al. Genome sequence of the vertebrate gut symbiont Lactobacillus reuteri ATCC 53608. J Bacteriol. 2011;193:4015-6.

[55] Rosander A, Connolly E, Roos S. Removal of antibiotic resistance genecarrying plasmids from Lactobacillus reuteri ATCC 55730 and characterization of the resulting daughter strain, L. reuteri DSM 17938. Appl Environ Microbiol. 2008; 74:6032-40.

[56] van de Guchte M, Serror P, Chervaux C, Smokvina T, Ehrlich SD, Maguin E. Stress responses in lactic acid bacteria. Antonie Van Leeuwenhoek. 2002;82:187-216.

[57] Liu XT, Hou CL, Zhang J, Zeng XF, Qiao SY. Fermentation conditions influence the fatty acid composition of the membranes of Lactobacillus reuteri 15007 and its survival following freeze-drying. Lett Appl Microbiol. 2014;59:398-403.

[58] Lebeer S, Vanderleyden J, De Keersmaecker SC. Genes and molecules of lactobacilli supporting probiotic action. Microbiol Mol Biol Rev. 2008;72:728-64.

[59] Bian L. An in vitro antimicrobial and safety study of Lactobacillus reuteri DPC16 for validation of probiotic concept [Master's tesis]. Massey University; 2008.

[60] Morita H, Toh H, Fukuda S, Horikawa $\mathrm{H}$ et al. Comparative genome analysis of Lactobacillus reuter and Lactobacillus fermentum reveal a genomic island for reuterin and cobalamin production. DNA Res. 2008; 15:151-61.

[61] Gänzle MG, Höltzel A, Walter J, Jung G, Hammes WP. Characterization of reutericyclin produced by Lactobacillus reuteri LTH2584. Appl Environ Microbiol. 2000; 66:4325-33.

[62] Mukai T, Asasaka T, Sato E, Mori K, Matsumoto M, Ohori H. Inhibition of binding of Helicobacter pylori to the glycolipid receptors by probiotic Lactobacillus reuteri. FEMS Immunol Med Microbiol. 2002;32:10510.

[63] Taranto MP, Vera JL, Hugenholtz J, De Valdez GF, Sesma F. Lactobacillus reuteri CRL1098 produces cobalamin. J Bacteriol. 2003;185:5643-7.

[64] Wang AN, Yi XW, Yu HF, Dong B, Qiao SY. Free radical scavenging activity of Lactobacillus fermentum in vitro and its antioxidative effect on growingfinishing pigs. J Appl Microbiol. 2009;107:1140-8.

[65] Hoffmann M, Rath E, Holzlwimmer $G$ et al. Lactobacillus reuteri 100-23 transiently activates intestinal epithelial cells of mice that have a complex microbiota during early stages of colonization. J Nutr. 2008;138:1684-91.

[66] Dicksved J, Schreiber O, Willing B et al. Lactobacillus reuteri maintains a functional mucosal barrier during DSS treatment despite mucus layer dysfunction. PLoS One. 2012;7:46399.

[67] Martinez RC, Seney SL, Summers KL, Nomizo A, De Martinis EC, Reid G. Effect of Lactobacillus rhamnosus GR-1 and Lactobacillus reuteri RC-14 on the ability of Candida albicans to infect cells and induce inflammation. Microbiol Immunol. 2009;53:487-95. 